\title{
Characterisation of DNA methylation machinery in human skin
}

\section{Bethany M Barnes ${ }^{1,2}$, David A Gunn³, Christopher EM Griffiths ${ }^{1,2}$ and Rachel EB Watson ${ }^{1,2}$}

${ }^{1}$ Centre for Dermatology Research, Faculty of Biology, Medicine and Health, The University of Manchester \& Salford Royal NHS Foundation Trust, Manchester Academic Health Science Centre, Manchester, UK

\section{Introduction}

- DNA methylation is an epigenetic mark usually associated with gene silencing, commonly referred to as switching genes 'on' or 'off.'

- The methylation state is influenced by 'writer' DNA methyltransferases (DNMTs) and the putative 'eraser' ten-eleven translocases (TETs) (fig 1).

- DNA methylation machinery in the skin remains to be characterised, and may influence appropriate epidermal stratification.

We sought to characterise the epidermal distribution and subcellular localisation of DNA methylation machinery in healthy human skin and in a cellular model of normal epidermal stratification.
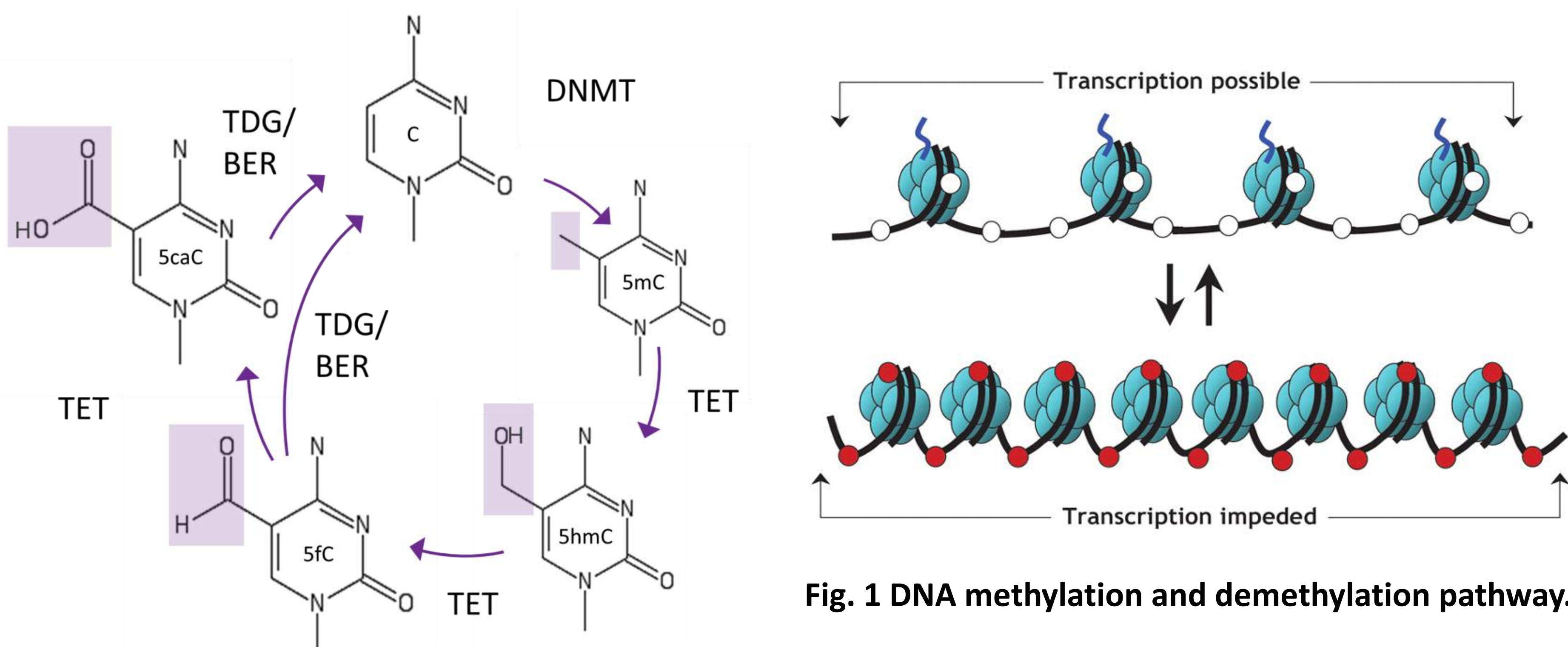

Fig. 1 DNA methylation and demethylation pathway.

\section{Methodology}

- Skin acquired via The University of Manchester Skin Health Biobank (MSHB) was analysed for DNMT and TET expression by immunohistological techniques.

- Primary keratinocytes grown to confluence were induced to differentiate via addition of $1.8 \mathrm{mM} \mathrm{CaCl}{ }_{2}$ ('calcium switching') and analysed by immunofluorescence and QPCR for DNMT expression.

\section{Methylation machinery is differentially distributed in human skin}

- Our staining confirmed the expression pattern of DNMT1 (fig $2 \mathrm{a}, \mathrm{b}$ ) previously reported by Sen et al. (2010) within the basal layer of the epidermis with limited expression in the outer, differentiated layers.

- DNMT3B is expressed ubiquitously across the epidermis in distinct foci overlapping with nuclei (fig $2 c, d$ ).

- Intense cytosolic immunoreactivity for DNMT3A of epidermal basal cells with diffuse nuclear staining seen in outer differentiated layers (fig $2 e, f$ ).
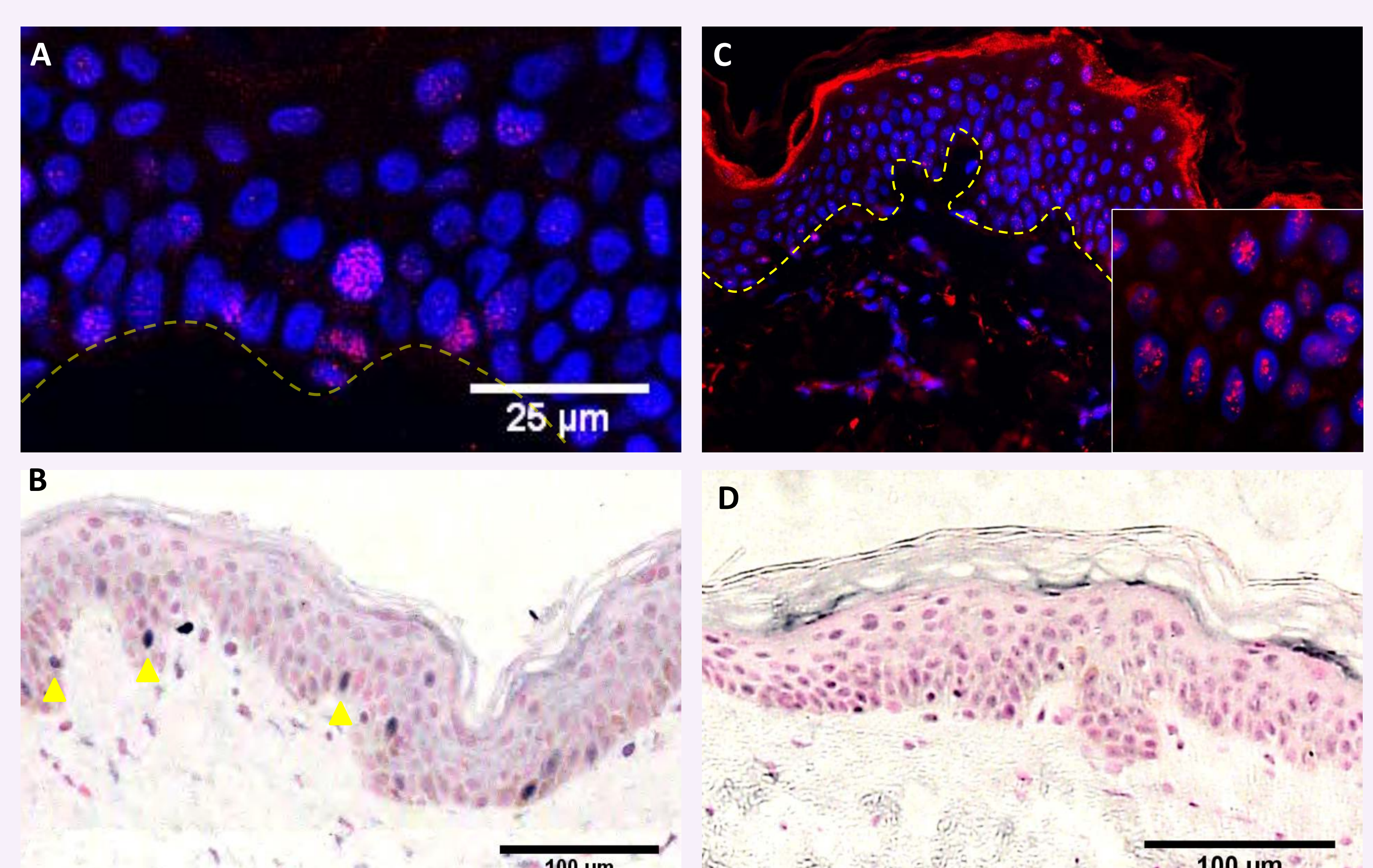

D

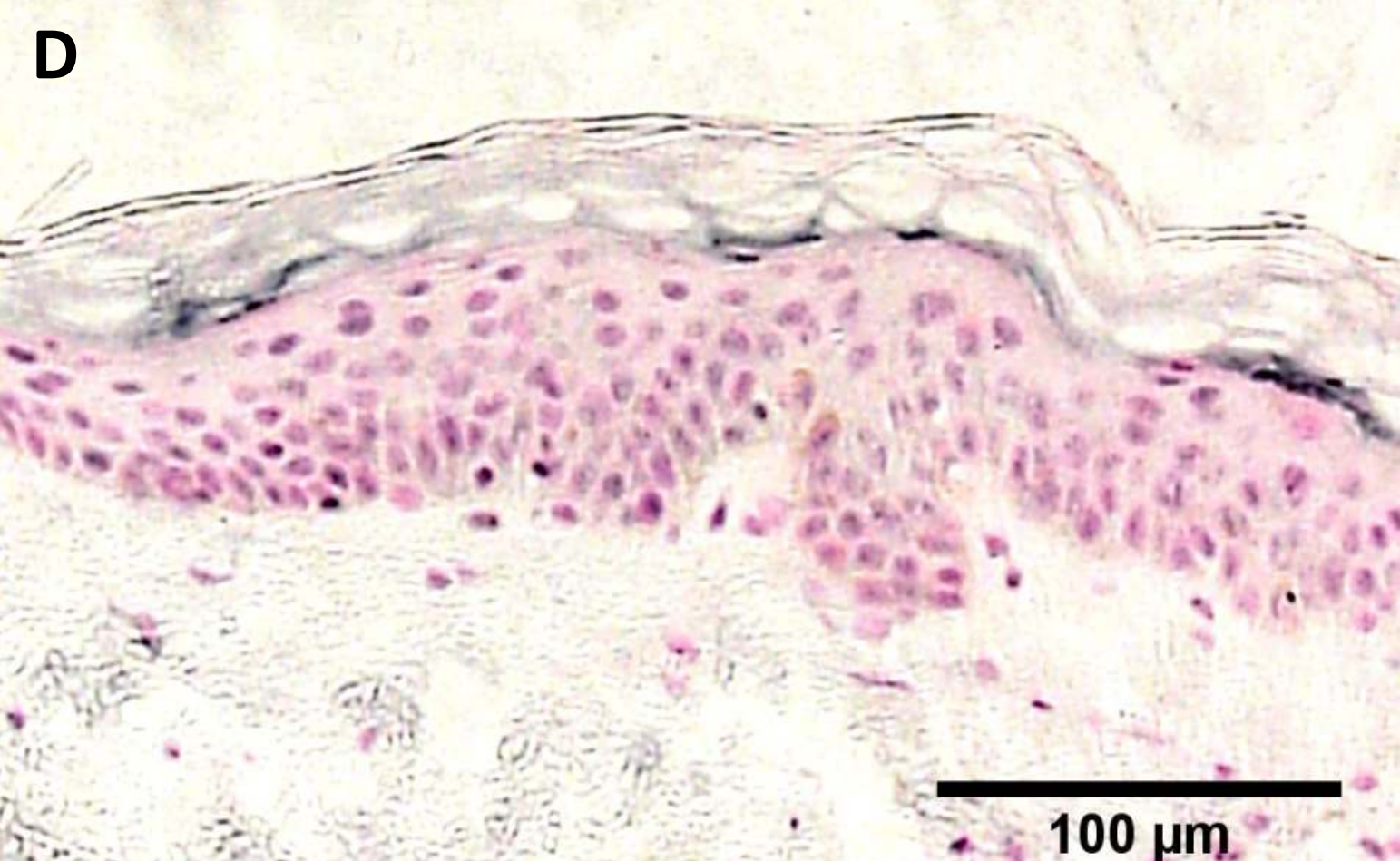

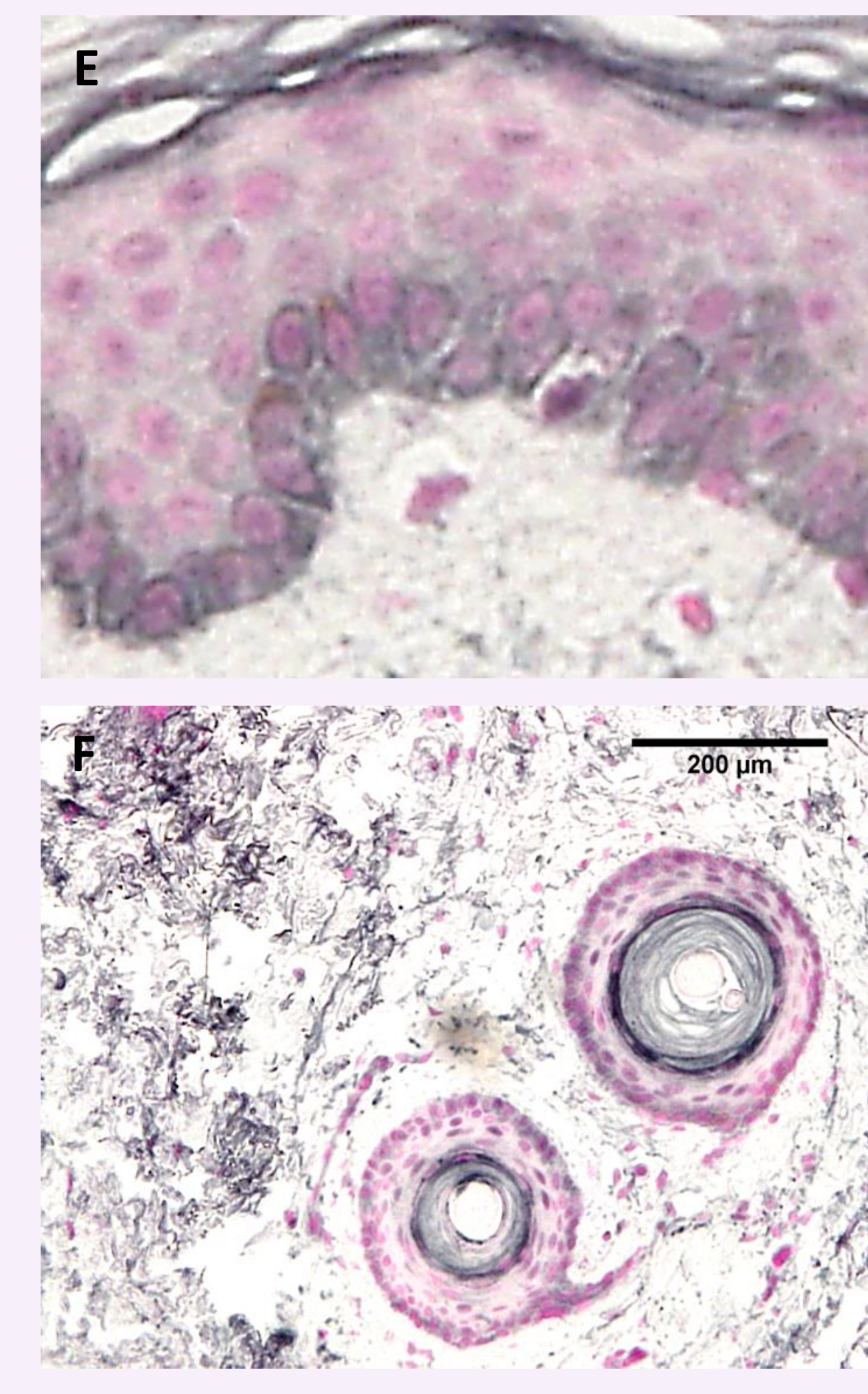

Fig. 2 DNA methyltransferase protein distribution in adult human abdominal skin. Dashed line represents the dermal-epidermal junction, arrows indicate immunoreactivity.

\section{Calcium switching shows concordant regulation of methylation machinery}

- Immunufluorescence analysis of primary human keratinocytes confirms immunoreactivity in the stratum basale of human skin (fig 3).
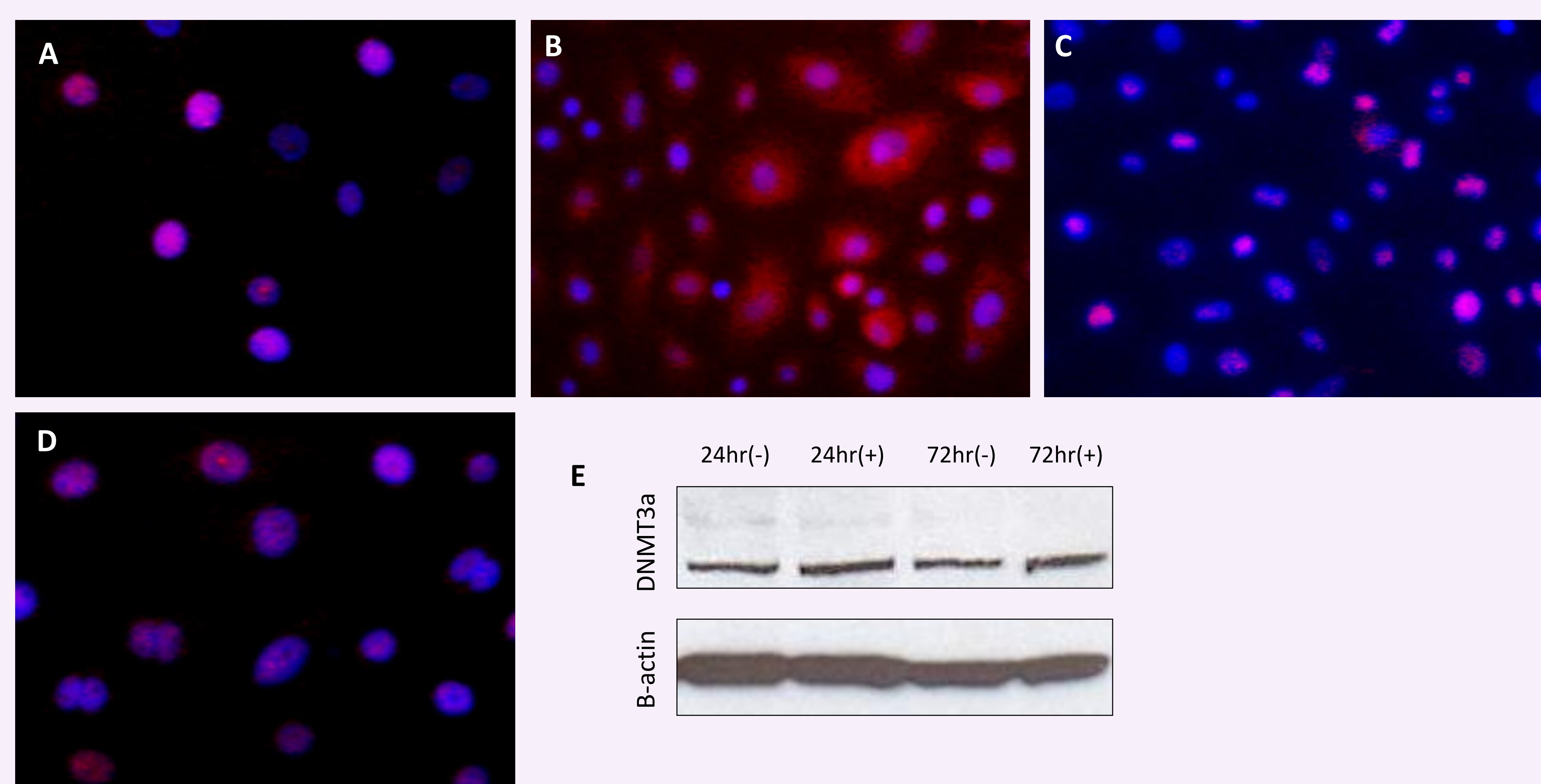

\section{E}

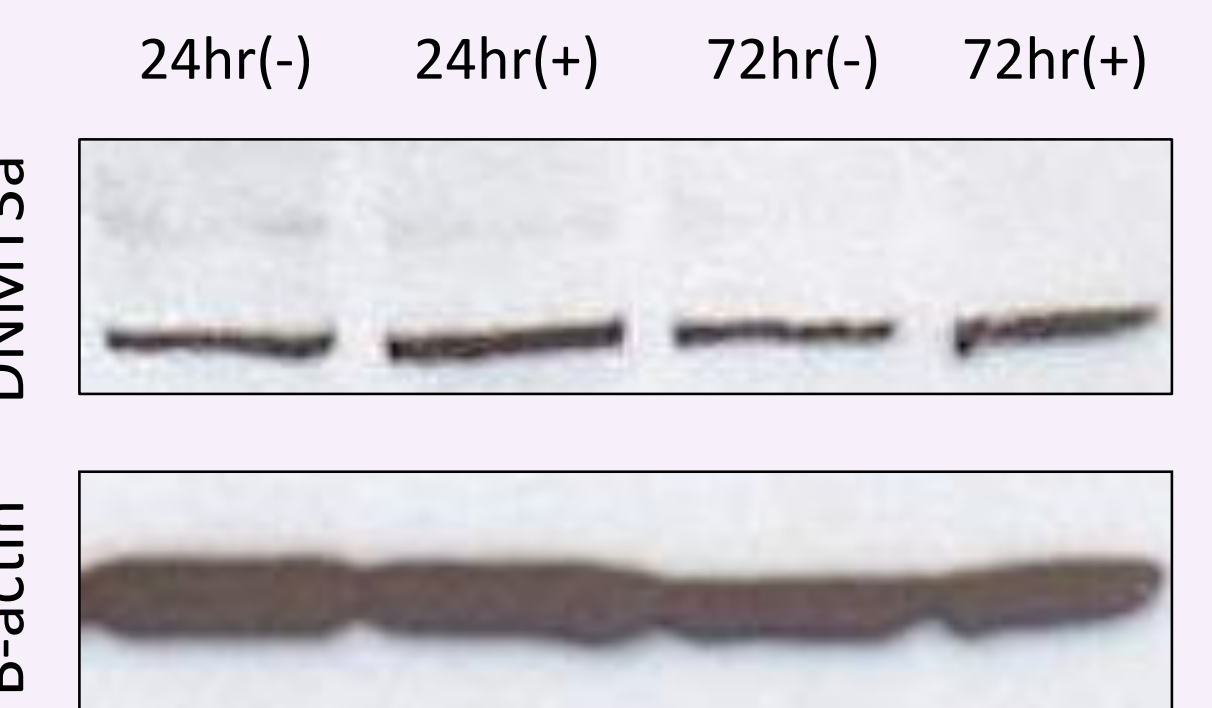

Fig. 7 Protein expression analysis of DNA methyltransferases in primary human keratinocytes. Immunofluorescence analysis of DNMT1 (a), DNMT3a (b), DNMT3b (c) and TET1 (d) Western blotting analysis of DNMT3a shows ablation of higher molecular weight band upon differentiation (e).
- Calcium switching cells down regulated DNMT1 and DNMT3b expression, while DNMT3a was reduced slightly but was not significant (fig 4).

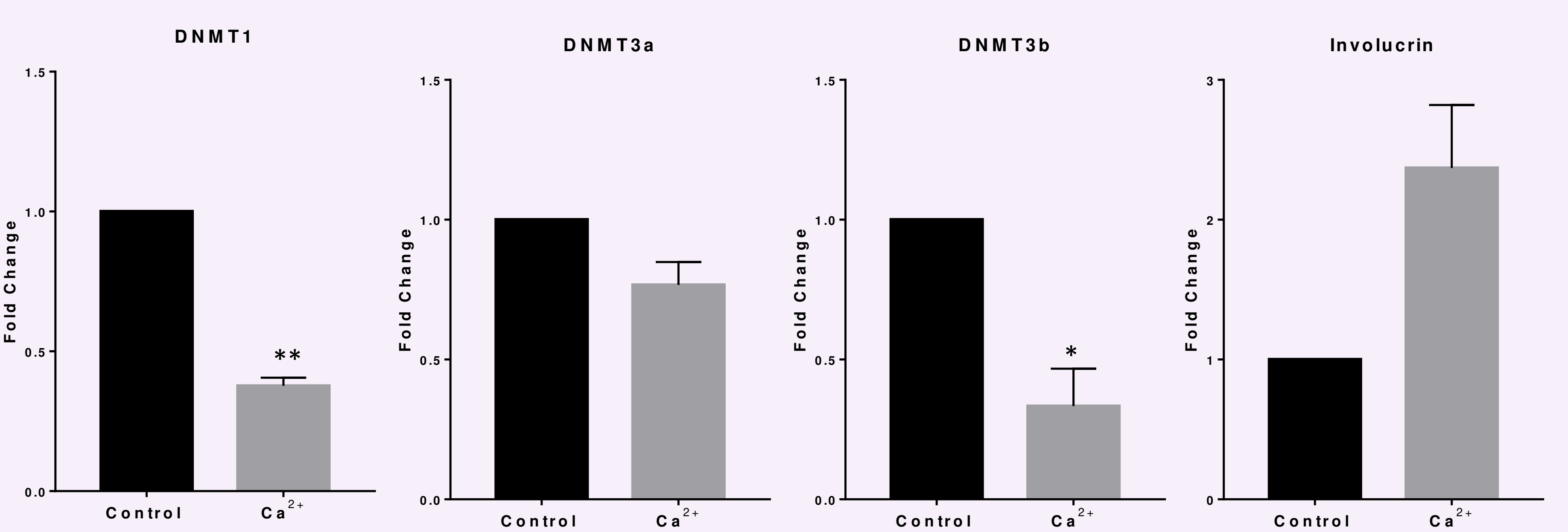

Fig. 6 qPCR analyses for DNA methyltransferases in non-differentiated and $\mathrm{Ca}^{2+}$ differentiated primary keratinocytes.

mRNA expression fold-change before (left) and after (right) differentiation. Differentiation was confirmed by upregulation of involucrin transcripts. Genes were normalised to peptidylprolyl isomerase A (PPIA). The bar indicates mean \pm SEM; * One-Sample T-Test: $\mathrm{P}<$ $0.05 * * *$ One-Sample T-Test: $\mathrm{P}<0.05$ 